Supporting information

\title{
Paper microfluidics and tailored gold nanoparticles for non- enzymatic, colorimetric multiplex biomarker detection
}

Tomás Pinheiro ${ }^{1}$, Ana C. Marques ${ }^{1}$, Patrícia Carvalho ${ }^{2}$, Rodrigo Martins ${ }^{1}$ and Elvira Fortunato $1,5,^{*}$

${ }^{1}$ CENIMAT|i3N, Departamento de Ciência de Materiais, Faculdade de Ciências e Tecnologia, Universidade NOVA de Lisboa and CEMOP/UNINOVA, Campus da Caparica, 2829-516 Caparica - Portugal

${ }^{2}$ SINTEF Materials and Chemistry, PB 124, Blindern, NO-0314, Oslo, Norway

${ }^{4}$ Lead Contact

*Correspondence: emf@fct.unl.pt 


\section{EXPERIMENTAL PROCEDURES}

\section{Synthesis of glucose-capped AuNPs}

Firstly, D-glucose solutions $(1.25-50 \mathrm{mM}$ ) are added in $500 \mu \mathrm{L}$ aliquots to eppendorf tubes (or microcentrifuge tubes), and placed on a vortex mixer (VELP Scientifica, Usmate, MB, Italy). While vortexing, $10 \mu \mathrm{L}$ of the $1 \mathrm{M} \mathrm{NaOH}$ solution are added to each tube. After approximately 10 seconds, $500 \mu \mathrm{L}$ of the $2.0 \times 10^{-4} \mathrm{M} \mathrm{HAuCl} 4$ solution are added to the vortexing solution and after a duration of about 5 to 80 seconds (depending on glucose concentration) a color change occurred, indicating the completion of the reaction and the formation of gold colloids. Agitation by vortexing was then continued for 5-10 seconds after color change to ensure reaction completion.

The synthesis of AuNPs on paper via glucose was reproduced based on the synthesis of AuNPs in solution previously described, altering the order of introduction of the reagents into the reaction system. Each well was impregnated with $2.5 \mu \mathrm{L}$ of the $1 \times 10^{-2} \mathrm{M} \mathrm{HAuCl} 4$ solution and the papers were left to dry at room temperature for about $10 \mathrm{~min}$. Then $2.5 \mu \mathrm{L}$ of $\mathrm{NaOH}$ solution is added to each well and allowed to dry at room temperature for $10 \mathrm{~min}$. Finally, $2.5 \mu \mathrm{L}$ of D-Glucose solution with desired concentration is added to each well and the microplates were left to dry at room temperature for a few seconds, until the formation of color signals. The drying process was continued for a few more minutes to ensure reaction completion.

\section{Synthesis of 2-TU aggregated AuNPs}

Typically, $80 \mu \mathrm{L}$ of an aqueous solution of HAuCl4 $(30 \mathrm{mM})$ is added to $2.8 \mathrm{~mL}$ of deionized water, under strong magnetic stirring. Posteriorly, $150 \mu \mathrm{L}$ of NaBH4 $(22 \mathrm{mM})$ aqueous solution is added to the mixture, to reduce the gold salt. The stirring is prolonged for 10 minutes, to promote a complete reduction of the gold salt by $\mathrm{NaBH} 4$, resulting in a ruby red colloidal solution. Posteriorly, $90 \mu \mathrm{L} \mathrm{2-TU}$ aqueous solution is added, to promote aggregation of AuNPs. The interaction between 2-TU aggregated AuNPs and UA was performed as follows: equal volumes of AuNP colloidal mixture and UA solution of desired concentration are added to an eppendorf under slight agitation at a vortex mixer.

2-TU aggregated AuNPs are used in the paper substrate as gold nanoprobes, by pipetting $50 \mu \mathrm{L}$ of colloidal gold mixtures into test zones. The colloidal gold mixture drop is left to dry over a hot plate at $50{ }^{\circ} \mathrm{C}$. When dried, the paper substrate is functionalized as a gold-on-paper platform, tested by deposition of a $10 \mu \mathrm{L}$ UA solution drop of known concentration, causing a color change in the paper platform.

\section{Digitonin functionalized AuNP synthesis:}

Reagents: Tetrachoroauric acid tryhydrate $\left(\mathrm{HAuCl}_{4} 3 \mathrm{H}_{2} \mathrm{O}\right)$; Sodium citrate; polyoxyethylene (20) sorbitan monolaurate (Tween 20); Sodium phosphate monobasic and dibasic; 16mercaptohexadecanoic acid (16-MHDA); sulfuric acid; digitonin.

Procedure:

A- Turkevich method for AuNPs synthesis: 
1) Prepare $95 \mathrm{~mL}$ of $\mathrm{HAuCl}_{4}$ solution containing $5 \mathrm{mg}$ of $\mathrm{Au}$; prepare $5 \mathrm{~mL}$ of $1 \%$ sodium citrate solution; All solutions are prepared using deionized water;

2) $\mathrm{Boil}_{\mathrm{HAuCl}}$ solution, until it reaches temperature close to $100{ }^{\circ} \mathrm{C}$; add sodium citrate solution aand let the reduction reaction occur for at least 30 minutes. Resulting solution presents a bright red coloration.

3) Let the solution cool at room temperature. When cooled, store in dark environment, at $4{ }^{\circ} \mathrm{C}$.

B- AuNP surface modification (thiol chemisorption):

1) Prepare $7 \mathrm{pH}, 10 \mathrm{mM}$ sodium phosphate buffer solution; Prepare $1.83 \mathrm{mg} / \mathrm{mL}$ Tween20 solution in sodium phosphate buffer; Prepare $0.5 \mathrm{mM}$ 16-MHDA solution in ethanol;

2) Add equal volumes ( $1 \mathrm{~mL})$ of AuNP colloidal solution obtained in (A) and Tween 20 solution into a glass flask, under light stirring (around $300 \mathrm{rpm}$ ) and let the mixture react for 30 minutes;

3) Introduce $1 \mathrm{~mL}$ (equal volume of the aforementioned solutions) of 16-MHDA solution to the reaction mixture and let the chemisorption occur for 3 hours;

4) Remove excess thiols and Tween 20 by repeated centrifugation (deposition of AuNPs at the bottom of the tube, followed by decantation of supernatants and resuspension in sodium phosphate buffer);

5) Store the resulting solution in dark environment, at $4{ }^{\circ} \mathrm{C}$, to prevent light induced flocculation.

C- Digitonin functionalization

1) Treat $2 \mathrm{~mL}$ of surface modified AuNPs (obtained in B) with digitonin, in the presence of conc. $\mathrm{H}_{2} \mathrm{SO}_{4}(0.5 \mathrm{~g})$, and stir the mixture for 5 hours, to allow for the esterification reaction to occur.

2) Purify the mixture by repeated centrifugation as in (B-4);

3) Store the resulting solution in dark environment, at $4{ }^{\circ} \mathrm{C}$, to prevent light induced flocculation.

D- Interaction between digitonin functionalized AuNPs (DAuNPs) and cholesterol

1) Prepare a stock solution of cholesterol with known concentration in a water acetone mixture $(50 / 50 \mathrm{v} / \mathrm{v})$;

2) Introduce $1 \mathrm{~mL}$ DAuNP solution to an Eppendorf tube and introduce $10 \mu \mathrm{L}$ cholesterol solution of known concentration; 


\section{AuNPs synthesis by direct glucose reduction of gold precursor}

Since the reduced gold is unstable in acid medium, $\mathrm{NaOH}$ was used to improve stability and promote hydrolysis of gold salt precursor (balanced chemical equation for the reduction is presented in SchemeS1).

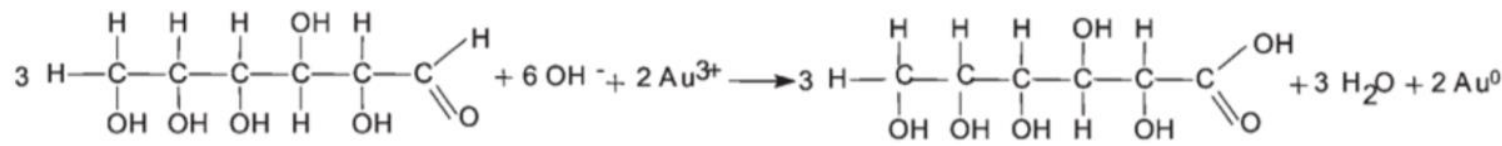

Scheme S1. Balanced chemical equation for reduction reaction of gold(III) ions by glucose, to form glucose-capped AuNPs and having gluconic acid as a product ${ }^{1}$.

Glucose standard solutions of increasing concentrations $(1.25-50 \mathrm{mM}$, including relevant physiological range from hypo to hyperglycemia) lead to variations in size of AuNPs, caused by the dynamics of the capping effect, as glucose binds to AuNPs' surface, preventing their aggregation. The first evidence of a blue shift in the LSPR peak (FigureS1) was obtained by the color succession of colloidal mixtures. For low glucose conditions, the solutions reveal a blue/purple color, whereas at higher glucose concentrations the solutions became pink-colored (Figure S1A). This trend is related to LSPR shift to lower resonance wavelengths with increasing glucose concentration, as showed by results from UV-Vis spectrophotometry in FigureS1A. The LSPR peak fluctuates between $530 \mathrm{~nm}$ (for $50 \mathrm{mM}$ glucose concentration) and $580 \mathrm{~nm}$ (for 1.25 $\mathrm{mM}$ glucose concentration. The plotting of LSPR peak $v s$. glucose concentration shows larger LSPR shifts for low glucose conditions (below $10 \mathrm{mM}$ ). Moreover, changes in plasmon resonance above $20 \mathrm{mM}$ were negligible, revealing a saturation behavior, caused by the limited surface area for small sized AuNPs to interact with remaining glucose, resulting in a decrease of assay sensitivity, showed in the plot of Figure S1B.

Results from TEM analysis provided information of size and morphology of nanoparticles for different glucose concentration conditions, confirming the size decrease of AuNPs. Images were obtained for three distinct glucose concentrations, $20 \mathrm{mM}$ (high concentration, Figure S1C), 10 $\mathrm{mM}$ (intermediate concentration, Figure S1D) and $2 \mathrm{mM}$ (low concentration Figure S1E). Results established that as glucose concentration rises, nanoparticles show increasingly symmetrical, regular and homogeneous shapes, besides a decrease in size $(3-20 \mathrm{~nm})$. For low glucose conditions, particles' size shows a significant increase $(50-100 \mathrm{~nm})$. The large nanoparticles formed at a low glucose concentration are apparently the result of a kinetic aggregation mechanism, since the amount of glucose involved on the synthesis is insufficient to cap the entire surface of the AuNPs, promoting their aggregation. On the other hand, a high amount of glucose, able to completely cap the nanoparticles surface, hinders their growth and aggregation, resulting in smaller AuNPs. Thus, the size difference between the AuNPs at different glucose concentrations correlates nicely with the observed changes in LSPR, previously reported. Furthermore, the capping effect of glucose towards particles can be observed in Figure S1E-inset and by elemental chemical analysis and mapping (Figure S1F-I), showing a complete and uniform distribution of 
$\mathrm{C}$ and $\mathrm{O}$ in particles surface, which are only introduced in the system by glucose. As it can be seen, the distribution of these two elements has higher concentrations at particles surface, covering it almost completely and guaranteeing particles' stability.
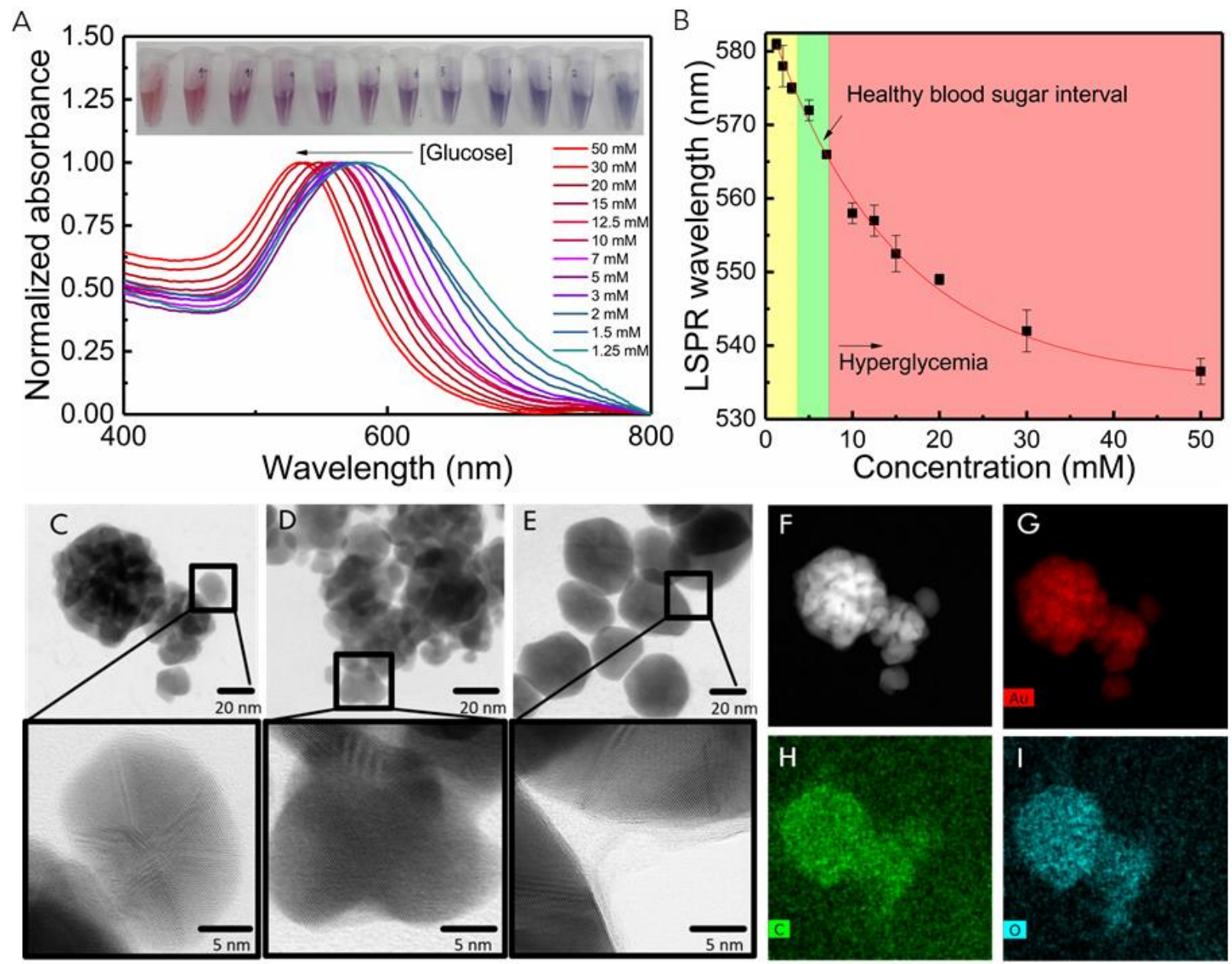

Figure S1. Plasmonic, size and morphological characteristics of colloidal gold nanoparticles mixtures by direct glucose reducing and capping. (A) Visual inspection results of colorimetric behavior of colloidal mixtures, showing a color succession from blue $(1.25 \mathrm{mM})$ to pink/red $(50 \mathrm{mM})$; Normalized absorbance spectra, showing the characteristic plasmonic blue shift caused by increasing glucose concentrations. (B)Plot of plasmonic resonance peak vs. glucose concentration, showing decreased sensitivity with increase of glucose concentration ( $n=3)$. (C) ABF/STEM image of AuNPs capped by $20 \mathrm{mM}$ of glucose;(D) ABF/STEM image of AuNPs capped by $10 \mathrm{mM}$ of glucose; (E) ABF/STEM image of AuNPs capped by $2 \mathrm{mM}$ of glucose, where an organic layer can be seen, to glucose capping; (F-I) HAADF/STEM image and corresponding EDS maps of AuNPs, showing the distribution of $\mathrm{Au}, \mathrm{C}$ and $\mathrm{O}$. The maps confirm the carbon and oxygen-based capping of particles, with uniform distribution of these elements at particles' surface. 
Results for the specificity of the chemical reaction towards the target analyte against common reducing molecules present in blood (fructose, galactose, glutathione, lipoic acid and ascorbate) ${ }^{1}$ is presented in (Figure S2).

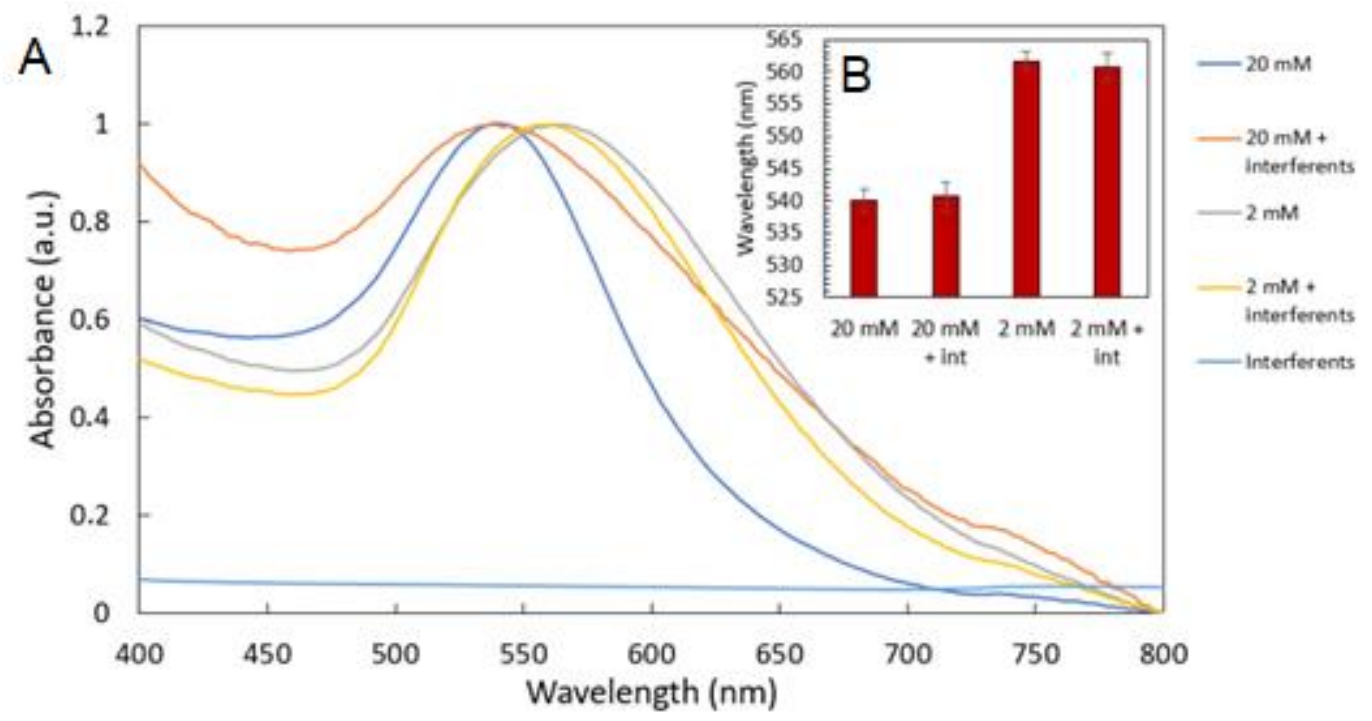

Figure S2. Specificity of the glucose-capped AuNPs assay, by means of interference testing. (A) Absorbance spectra of glucose capped AuNPs synthesized using two glucose concentrations ( 2 and $20 \mathrm{mM}$ ), in the presence and absence of interfering agents and using a solution containing only the interfering agents. (B) Histogram of LSPR wavelength vs. synthesis conditions, showing that the presence of interfering agents does not significantly influence the LSPR wavelengths $(n=3)$.

\section{In-situ synthesis of glucose capped AuNPs in paper substrate for glucose measuring}

In the paper assay for glucose sensing, the reduction of $\mathrm{HAuCl}_{4}$ is performed directly in the paper substrate, by consecutive deposition and drying of reagents. Firstly, a drop of gold salt aqueous solutions is deposited and dried, giving the paper test zone a yellow hue. Secondly, hydrolysis is carried by deposition of $\mathrm{NaOH}$ solution on top of the gold salt, resulting in loss of the yellow color characteristic of gold, but without reduction and production of AuNPs. We found that the final addition of glucose standard solution posteriorly to the addition of these reagents leads to the synthesis of AuNPs directly in the paper substrate, with plasmonic, colorimetric characteristics dependent on reaction parameters. AuNPs directly synthesized in the paper substrate show a pink coloration, with rising color intensity correlated to increasing glucose concentrations. This color intensity is apparently explained by the increasing amounts of AuNPs being formed in the paper substrate.

We found that gold salt and $\mathrm{NaOH}$ concentration influence the hue and intensity of color signals. The introduction of increasing amounts of gold salt into the paper substrate leads to the appearance of more intense coloration in the test zones, as more quantities of gold salt leads to the synthesis of more AuNPs. Also, there is a change in the hue of AuNPs formed in the paper substrate. This can be seen by comparison of, where gold salt concentration was changed from 10 to $30 \mathrm{mM}$. When increasing amounts of $\mathrm{NaOH}$ are introduced, hue and color intensity of AuNPs also changes, as $\mathrm{NaOH}$ speeds the kinetic process of reduction and capping of nanoparticles, forming more AuNPs at a more rapid pace. The differences in color signals of the assay for different $\mathrm{NaOH}$ concentrations can be seen in, where $\mathrm{NaOH}$ concentrations go from 1 to $3 \mathrm{M}$, respectively. Also, $\mathrm{NaOH}$ influences the sensitivity range of the assay, as the amount of glucose necessary for AuNPs' 
formation in paper changes with the increase of this precursor reagent. Results are presented in Figure 2 in the manuscript.

In comparison to the colorimetric behavior of glucose capped AuNPs in colloidal mixtures, AuNPs produced in paper do not show such a pronounced dynamic range of resonance. This is because instead of undergoing Brownian motion when in fluid medium, reagents are immobilized in paper fibers. This may hinder the reduction and capping effects of glucose to a more localized fashion, thus decreasing the size range when comparing AuNPs formed in the fluid medium and in the paper substrate, for the same glucose concentration span. Parameter optimization was performed, mainly by achieving the optimal gold salt and $\mathrm{NaOH}$ concentrations, to attain the needed requirements of color uniformity, reproducibility and sensitivity, resulting in optimal

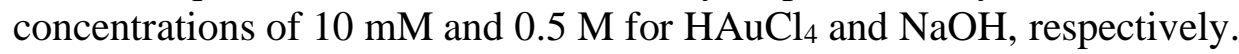

Specificity of the paper-based assay was tested, with resulting showing that common reducing molecules do not influence the R/G ratio. Besides, the considered interfering agents do not cause the formation of AuNPs in the paper substrate independently, resulting in a good specificity for the assay (Figure S3).

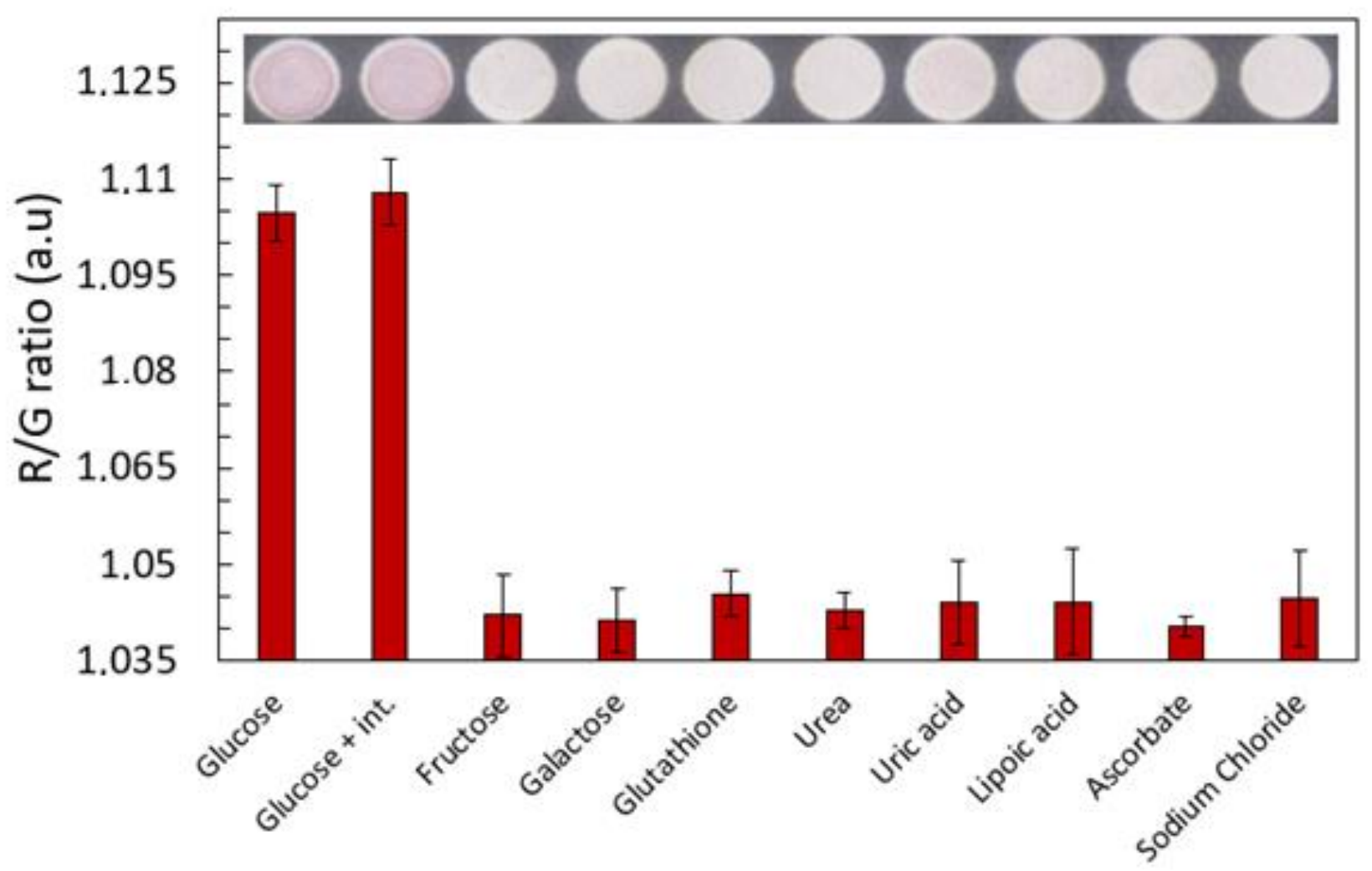

Figure S3. Specificity of paper-based glucose assay, by means of interference testing. Results show that the presence of interfering agents do not significantly influence the Red/Green ratio values used to describe the color of directly synthesized AuNPs. Furthermore, the considered metabolites do not alone, cause reduction of gold salt directly in the paper substrate (color signal obtained using $5 \mathrm{mM}$ glucose)

AuNPs characterization was performed to better understand this in-situ synthesis. Through Surface-Enhanced Raman Spectroscopy (SERS), we showed that in-situ synthesized AuNPs lead to an enhancement of the Raman signal (using Rhodamine 6G as probe), when compared to the signal obtained for R6G on glass, resulting in SERS-active substrate. In this case, two 
characteristic lines at 1360 and $1510 \mathrm{~cm}^{-1}$, corresponding to the Raman vibrations associated with R6G were identified and used to calculate spectral intensity and respective enhancement factors (EF). EFs in the order of magnitude of $10^{2}$ were achieved for both characteristic peaks, showing the potentiality of these AuNPs as SERS probes, with further optimization in terms of AuNP distribution in the paper substrate (Figure S4). AuNPs synthesized with different glucose concentration conditions were used $(40,50,60 \mathrm{mM})$, showing that the capability for enhancing the Raman signal increases with the amount of glucose used for the synthesis (probably because more AuNPs are present), also indicating that an indirect assay, using R6G as a probe molecule, could be used for glucose level monitoring.

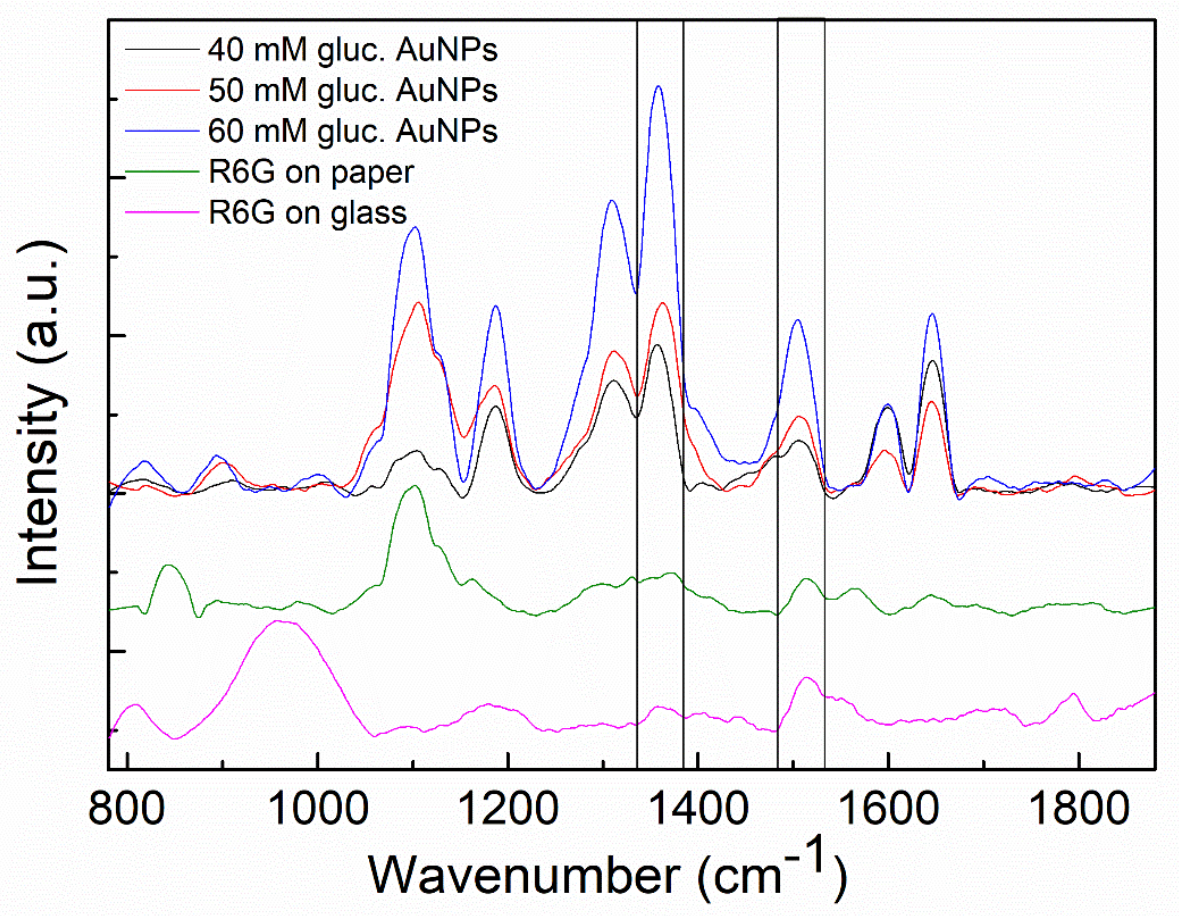

Figure S4. SERS analysis of paper substrates with directly produced AuNPs, with the presence of R6G. In comparison with individual presence both in glass and in paper as reference, there is an increase in the Raman signal for two characteristic peaks $(1360$ and $1510 \mathrm{~cm}-1)$, showing the potential of these AuNPs to be used as SERS probes.

Color signal variability after assay performance was assessed, by following the difference between grayscale values for the lowest $(1.25 \mathrm{mM})$ and highest $(20 \mathrm{mM})$ glucose concentration used for assay performance, showing how the evaporation of the aqueous sample matrix leads to a decrease in signal intensity and stabilization of this difference, that reduces the sensitivity range over time. Thus, 2 minutes scanning after assay performance was used. 


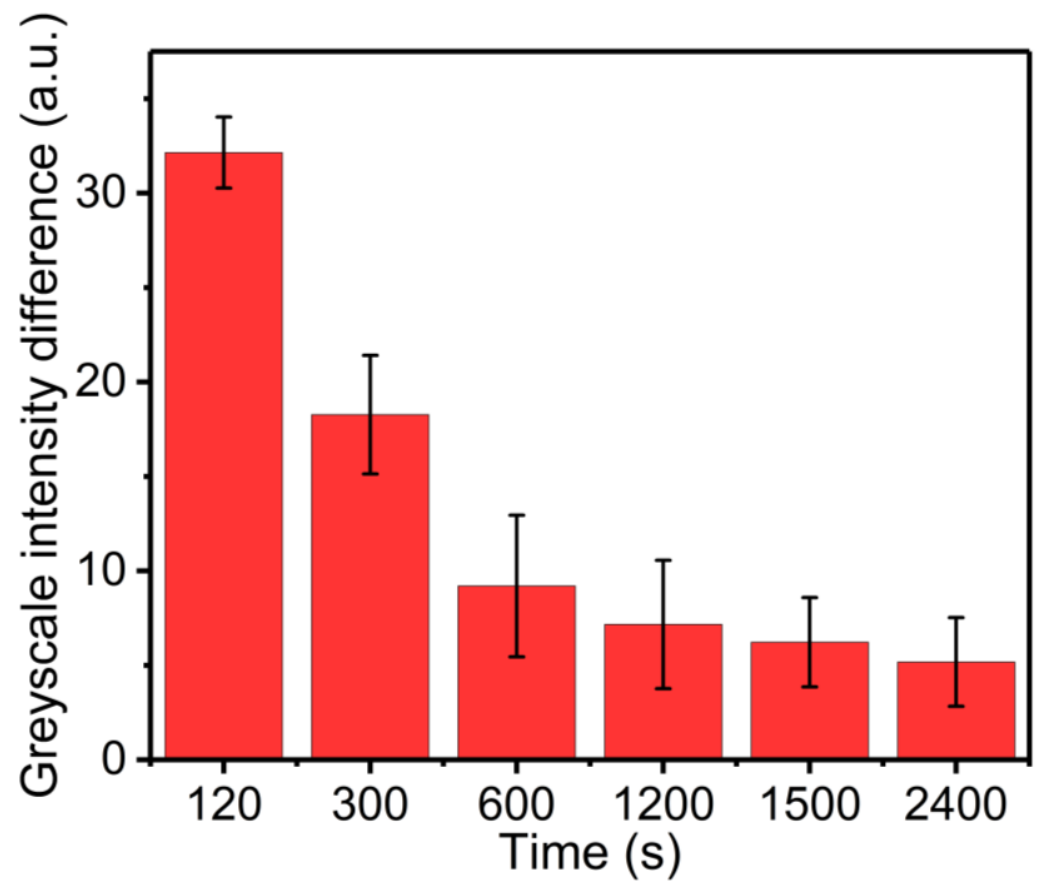

Figure S5. Testing of color signal stability over time, by monitoring the grayscale intensity difference for color signals obtained with 1.25 and $20 \mathrm{mM}$ )

Storage stability was assessed by maintaining paper-based platforms with immobilized gold salt in the dark at $4{ }^{\circ} \mathrm{C}$, and testing the in-situ synthesis after 3, 7, 10 and 20 days of storage. Results indicate that the capability for AuNPs synthesis is maintained, with the same trend of increase color intensity associated with increase of glucose concentration (1.5, 3, 10 and $15 \mathrm{mM})$. After 20 days of storage, the particle formation capability is maintained, with a slight decrease in overall color intensity (leading to increase of the grayscale values), probably due to some immobilized reagent degradation. However, the difference in grayscale value for the lower and higher glucose concentrations is maintained, even after this storage period. Thus, results indicate good storage resistance, that could be improved with more rigid storage protocols, using sealing and other strategies.

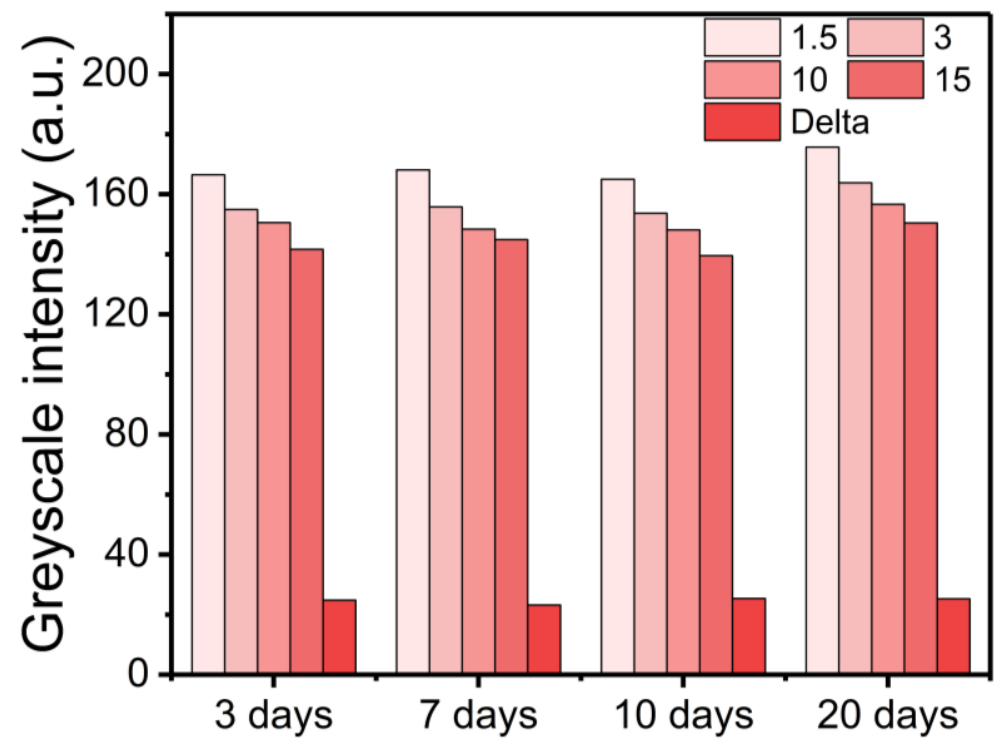

Figure S6. Storage stability testing of the in-situ synthesis AuNPs synthesis promoted by glucose 
Gold-on-paper uric acid assay using 2-TU surface modified AuNPs:

$2 \mathrm{HAuCl}_{4}+2 \mathrm{NaBH}_{4}+6 \mathrm{H}_{2} \mathrm{O} \longrightarrow 2 \mathrm{Au}^{0}+2 \mathrm{NaBO}_{3}+8 \mathrm{HCl}+7 \mathrm{H}_{2}$

Scheme S2. Balanced chemical equation for the reduction of gold(III) ions from the gold salt precursor by sodium borohydride, to form AuNPs $^{2}$. These AuNPs are subsequently modified at a surface level, by the thiol moiety in 2-thiouracil.

Uric acid assay performed with modified AuNPs using $0.1 \mathrm{mM}$ of 2-TU, showing the aggregation behavior of this plasmon coupling system, where the introduction of 2-TU into the reaction mixture leads to the formation of AuNP complexes with a characteristic plasmon resonance peak wavelength of $655 \mathrm{~nm}$. As it can be seen, colloidal mixtures evolve from a red coloration (low UA concentrations) to purple (mixture of non-aggregated AuNPs and aggregate complexes) and blue, with the appearance of a resonance peak around $655 \mathrm{~nm}$ in the absorbance spectra. This condition can be used to measure UA in the physiological range using colloidal gold solutions, by monitoring the ratio of absorbance at 655 and $530 \mathrm{~nm}$.
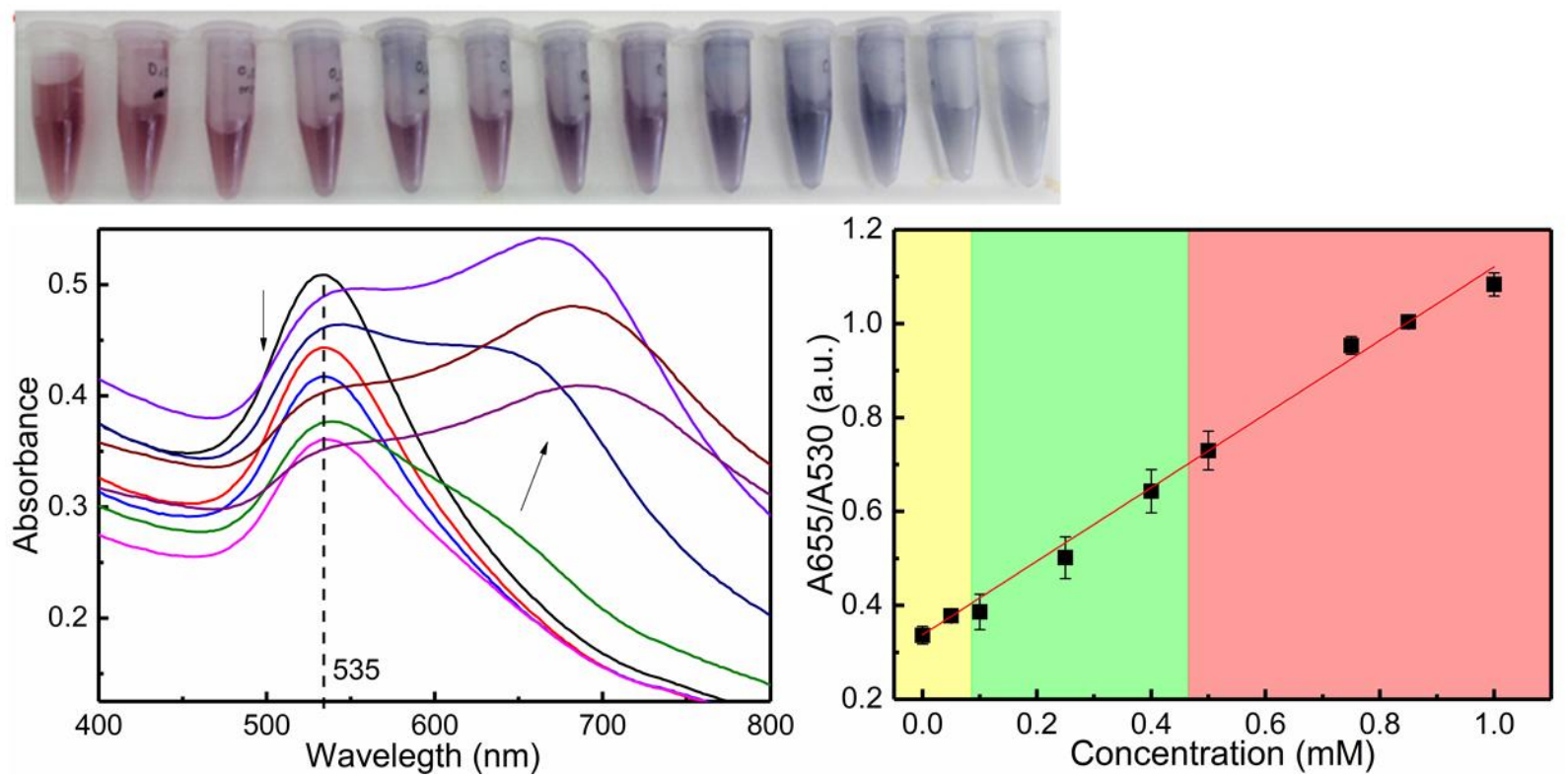

Figure S7. Aggregation assay of UA, using 0.1 mM 2-TU to surface modify AuNPs. (A) Visual inspection of colorimetric behavior of the assay, showing the change from red to blue as higher concentrations of UA are introduced into the system. (B) Absorbance spectra of colloidal gold mixtures presented in (A), showing that the original red colored AuNPs have a LSPR peak wavelength at around $530 \mathrm{~nm}$. This peak decreases in intensity for higher amount of UA introduced, with a new peak around $655 \mathrm{~nm}$ increasing its intensity.(C) Plot of LSPR absorbance vs. UA concentration showing the linear correlation for UA concentration from 0 to $1 \mathrm{mM} \mathrm{n}=3,=0.783+0.3376$ ) 
Specificity assays were also performed in the paper substrate for uric acid sensing quantification methods, showing the good specificity of the methods. The metabolites were introduced into the test zones with pre-deposited AuNPs, showing that they do not cause the etching or antiaggregation effect described for this assay, showing a good specificity of the method.

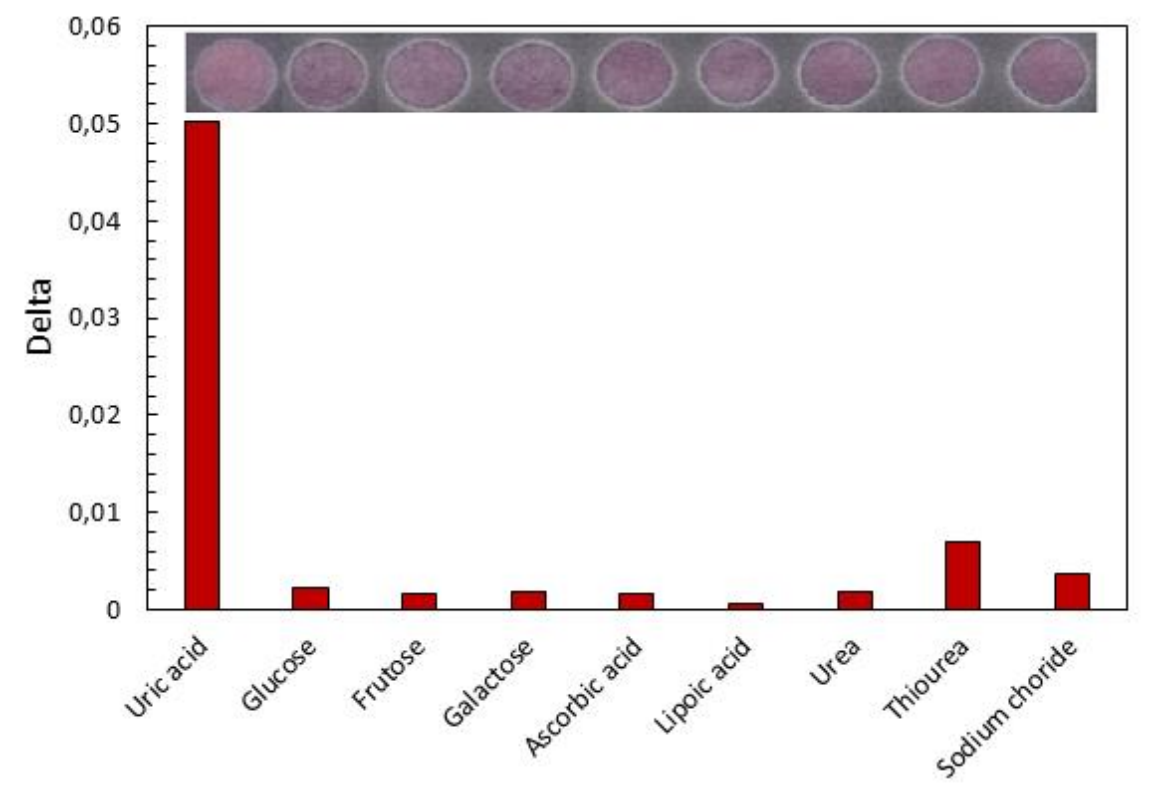

Figure S8. Specificity of paper-based UA assay, by means of interference testing. Results showed that the presence of interfering agents do not significantly influence the Red/Blue ratio values (Delta values describe the difference between blank result and the tested condition) used to describe the color obtained after reaction. Furthermore, the considered metabolites do not alone cause etching effects towards the deposited AuNPs.
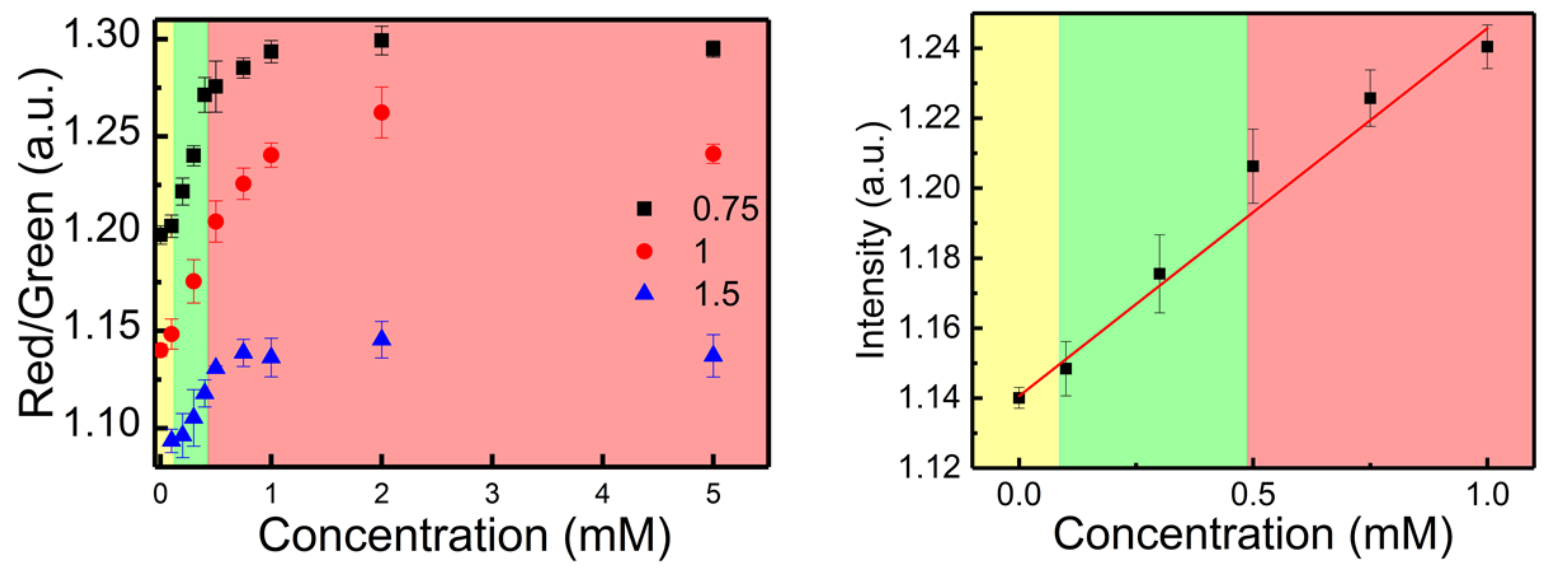

Figure S9. Alternative calibration metric based on the Red/Green channels ratio. This metric is used to monitor the colorimetric behavior of the assay, by establishing the variation of the red channel when compared to the green channel, which increases its intensity more rapidly than the green channel, with increase of UA levels.

Color signal variability after assay performance for 2-TU modified AuNPs immobilized at paper substrate was assessed, by following the difference between $\mathrm{R} / \mathrm{B}$ ratio values for the lowest $(0.1$ $\mathrm{mM})$ and highest $(1 \mathrm{mM})$ uric acid concentration used for assay performance, showing how the evaporation of the aqueous sample matrix leads to a decrease in signal intensity and stabilization 
of this difference, that reduces the sensitivity range over time. Thus, 2 minutes scanning after assay performance was used.

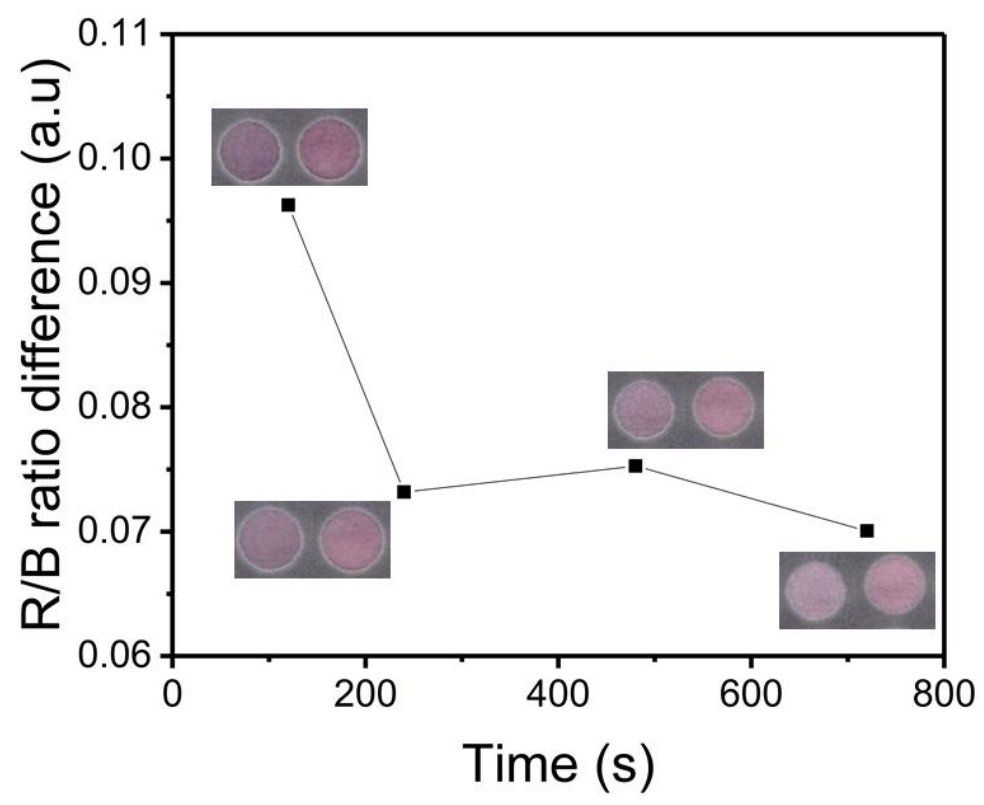

Figure S10. Variation of color signals for UA gold-on-paper assay over time after assay performance.

Testing of storage resistance of 2-TU modified AuNPs was performed, with results showing that up to 15 days, the characteristic blue-shift of particles' anti-aggregation effect towards UA introduction occurs, with color changing from blue to red for increased UA levels. A slight decrease of the red coloration can be seen after 10 days of storage, showing some degradation of the particles ability to respond to the introduction of UA.

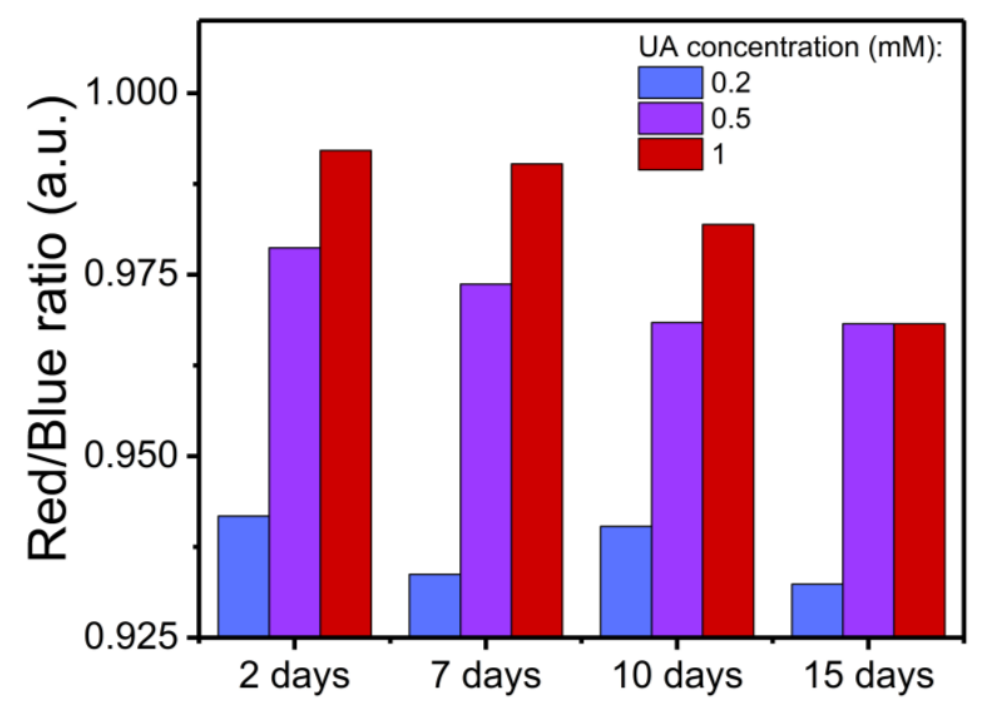

Figure S11. Storage resistance testing of 2-TU modified AuNPs at paper substrate. 
Gold-on-paper free cholesterol assay using digitonin modified AuNPs:

$2 \mathrm{AuCl}_{4}^{-}+3\left(\mathrm{CH}_{2} \mathrm{COOH}\right)_{2} \mathrm{C}(\mathrm{OH}) \mathrm{COO}^{-} \longrightarrow 2 \mathrm{Au}^{0}+8 \mathrm{Cl}^{-}+3\left(\mathrm{CH}_{3}\right)_{2} \mathrm{C}=\mathrm{O}+9 \mathrm{CO}_{2}+3 \mathrm{H}^{+}$

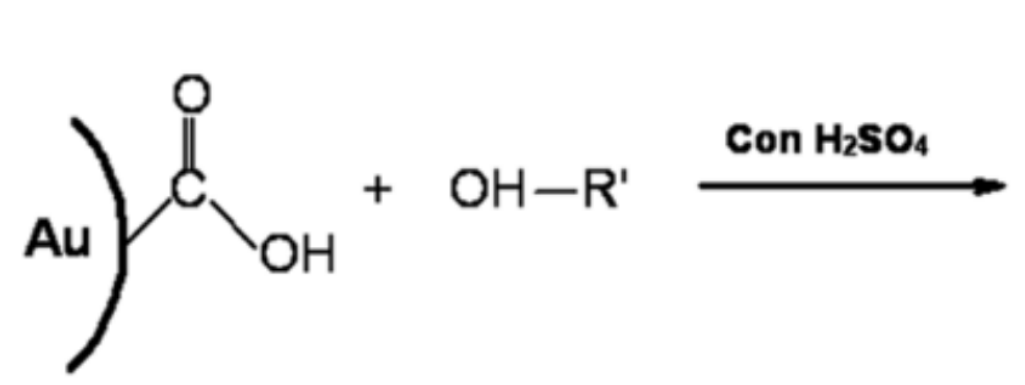

16-MHDA AuNP
Digitonin

Esterification Reaction

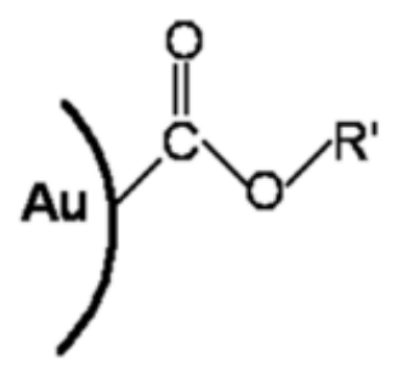

DAuNP

Scheme S3. Balanced chemical equation for the reduction of gold(III) ions from the gold salt precursor by sodium citrate, to form citrate-capped AuNPs ${ }^{3}$; These AuNPs are then modified at the surface level, by the thiol moiety of 16-MHDA, which is used to perform an esterification reaction establishing an ester bond to digitonin ${ }^{4}$ 
Microfluidic paper-based device architectures, operation and testing:

An alternative architecture for the 3D multiplex $\mu \mathrm{PAD}$ is presented and applied for the glucose insitu AuNPs synthesis assay. This device has an alternative microfluidic network design and test zone disposition, comprising of symmetric channels and reaction zones. In Figure S8A, device architecture and components are presented. This architecture presents the same components, (i) sample entrance zone, (ii) microfluidic network and (iii) test zones, however with alteration in network design and test zone disposition. In Figure S8B-C, glucose assay was performed using this architecture, with two different test zone diameters ( 3 and $6 \mathrm{~mm}$, respectively), showing that more uniform color signals are achieved. The corresponding plots of glucose concentration $v s$. calibration metrics are presented in Figure S8D-E, for the red channel and greyscale mean values, showing the decrease of these values correlated with increase in glucose concentration.
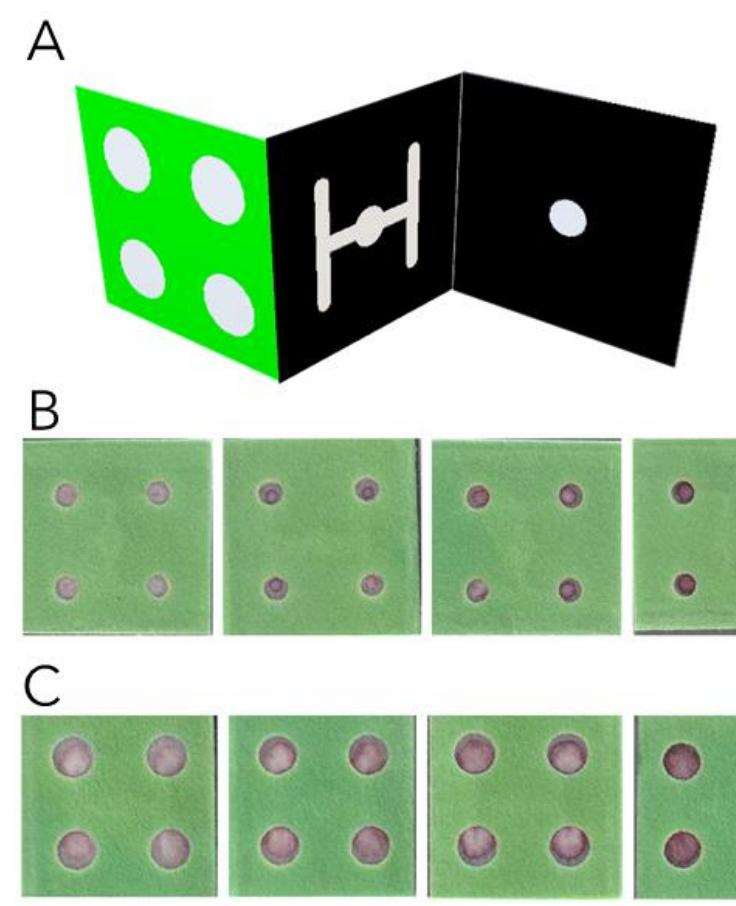

2

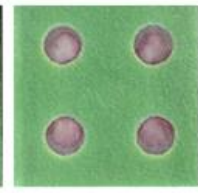

3

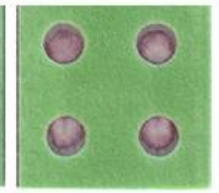

5
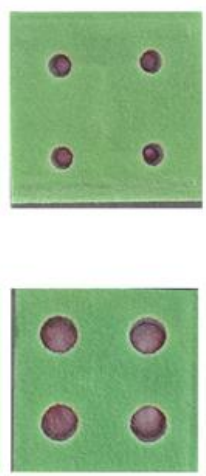

10
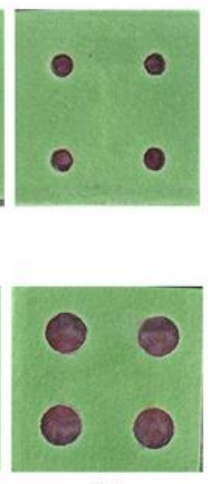

20
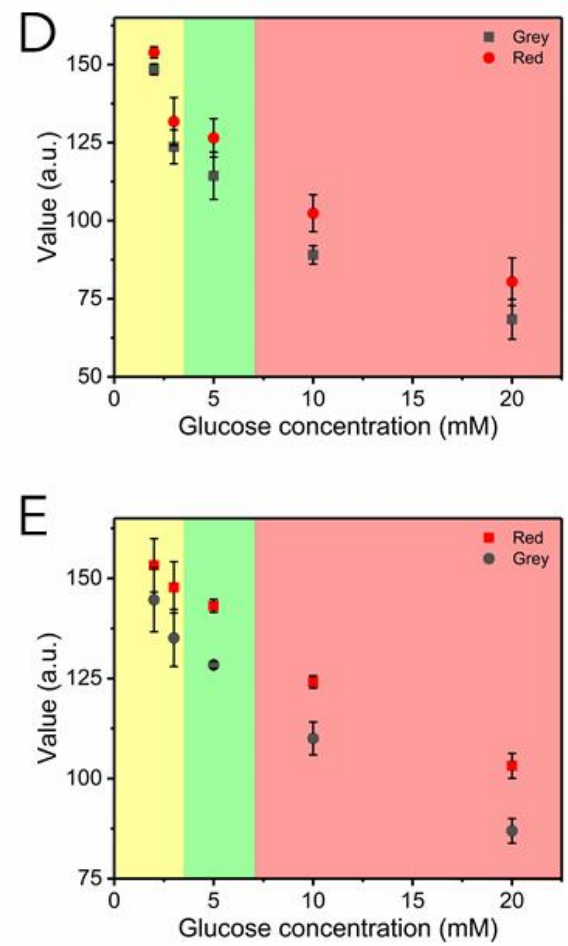

Figure S12. Alternative $\mu \mathrm{PAD}$ architecture applied for in-situ AuNPs synthesis glucose assay. (A) Device architecture and components. (B and C) color signals of glucose assay for test zones with 3 and $6 \mathrm{~mm}$, respectively. (D and $E$ ) plots of glucose concentration vs. red channel and greyscale mean values.

\section{References:}

1. Liu, J., Qin, G., Raveendran, P., \& Ikushima, Y. (2006). Facile "green" synthesis, characterization, and catalytic function of $\beta$-D-glucose-stabilized $\mathrm{Au}$ nanocrystals. Chemistry-A European Journal, 12(8), 2131-2138.

2. Wagner, J., Tshikhudo, T. R., \& Köhler, J. M. (2008). Microfluidic generation of metal nanoparticles by borohydride reduction. Chemical Engineering Journal, 135, S104-S109. 
3. Agunloye, E., Panariello, L., Gavriilidis, A., \& Mazzei, L. (2018). A model for the formation of gold nanoparticles in the citrate synthesis method. Chemical Engineering Science, 191, 318-331.

4. Raj, V., Jaime, R., Astruc, D., \& Sreenivasan, K. (2011). Detection of cholesterol by digitonin conjugated gold nanoparticles. Biosensors and Bioelectronics, 27(1), 197-200. 